\title{
Comparison of Polyclonal Antibody Sera for Early Prophylaxis following Cardiac Transplantation ${ }^{1}$
}

\author{
Steven F. Bolling, M.D., Mack C. Stirling, M.D., Paula Miska, R.N., and G. Michael Deeb, M.D. \\ Section of Thoracic Surgery, University of Michigan, Ann Arbor, Michigan 48109
}

Submitted for publication November 16, 1988

\begin{abstract}
In order to test different polyclonal antibody regimes as early prophylaxis against cardiac rejection, 42 patients (ages 30 to 60 years) transplanted at the University of Michigan from December 1986 to August 1988 were randomized to receive antithymocyte globulin (ATGAM, Upjohn, $n=19$ ) or antilymphoblast globulin (MALG, University of Minnesota, $n=23$ ). Cyclosporine (CYA), steroids, and azothiaprine (AZA) administration was similar in all randomized patients during early prophylaxis. CYA was begun preoperatively and maintained at a serum level of $250-300 \mathrm{ng} / \mathrm{ml}$. After an initial steroid taper, patients were maintained on $0.3 \mathrm{mg} / \mathrm{kg} / \mathrm{day}$. AZA was begun after polyclonal prophylaxis at $1-2 \mathrm{mg} / \mathrm{kg}$. All patients received either ATGAM or MALG for 7 days or until the serum CYA reached $250 \mathrm{ng} / \mathrm{ml}$. Although sex, pretransplant hemodynamics, follow-up length, total drug dose, mortality (one per group), postoperative white blood cell and lymphocyte counts did not differ between groups, MALG significantly delayed the first rejection episode as compared to ATGAM (35 \pm 4 vs $22 \pm 3$ days, $P<0.05)$. Additionally, there was decreased rejection during follow-up for the MALG group with $1.5 \pm 0.2$ rejections per patient as compared to $2.3 \pm 0.3$ with A'TGAM. Furthermore, the significant infection rate with MALG was only half that of the ATGAM group (6/23 vs 11/19) $(P<0.05)$. The beneficial effect of MALG may be due to immune-specific differences in its polyclonal spectrum. (c) 1989 Academic Press, Inc.
\end{abstract}

\section{INTRODUCTION}

With the onset of cyclosporine immunosuppressive therapy, cardiac transplantation has progressed from an experimental to a therapeutic procedure. The exponential increase in the number of patients receiving cardiac transplantation and the large patient population that has survived up to 5 years have made it apparent that high serum levels of cyclosporine can cause hypertension, progressive renal failure, and other untoward side effects.

\footnotetext{
${ }^{1}$ Presented at the Annual Meeting of the Association for Academic Surgery, Salt Lake City, UT, Nov. 16-19, 1988.
}

In an effort to obtain the maximum benefits of cyclosporine as an immunosuppressive agent, but minimize its potentially toxic side effects, combination immunosuppressive regimens, utilizing lower doses of cyclosporine, have been studied. Most combination regimens have included a lowered loading dose of cyclosporine. During the loading dose interval, protection against rejection is necessary for the heart, and antilymphocytic globulin used as early prophylaxis has been one method [5]. Although much clinical data on antithymocyte globulin (ATGAM) and antilymphoblast globulin (MALG, University of Minnesota) as antilymphocytic agents have accumulated, there has been no randomized prospective study comparing the two agents. This study randomized use of ATGAM or MALG in combination with tapering doses of steroids, azothiaprine, and cyclosporine, in 42 cardiac transplant patients.

\section{MATERIAL AND METHODS}

All patients receiving orthotopic heart transplantation at the University of Michigan between 30 and 60 years of age were eligible for this randomized prospective study. Between December 1986 and August 1988, 42 adult patients between the ages of 30 and 60 years were transplanted at the University of Michigan Medical Center, 23 of these patients were randomized to receive MALG prophylaxis therapy and 19 of them were randomized to receive ATGAM prophylaxis therapy. Patients above the age of 60 were excluded from this protocol, secondary to the possible immune tolerance seen in this age group. Patients below 30 years were also excluded because of the variable immune response seen in adolescents and young adults. All patients met the standard criteria for end-stage heart failure, requiring orthotopic heart transplantation.

At the time of their transplant, the patients were randomly allocated to ATGAM or MALG therapy, after informed consent was obtained. The study was performed in accordance with the ethical standards of the Institutional Review Board on Human Use of the University of Michigan. There were no parameters or conditions set to preferentially place a patient into either group. Unfortunately, optimal randomization was not obtained, sec- 
ondary to unavailability of a specific agent. However, the maximum number of patients to receive any given agent consecutively was four.

The immunosuppressive regimen consisted of MALG (15 mg/kg/day) or Upjohn ATGAM (10 mg/kg/day) in $500 \mathrm{cc}$ of normal saline, given immediately postoperatively in the intensive care unit and then daily for a total of seven doses, or until the serum cyclosporine level reached $250 \mathrm{ng} / \mathrm{ml}$. The doses were selected and used at the dose recommended by the manufacturer of each specific drug and are the currently clinically used doses. As was stated by Najarian et al. [10] during the development of Minnesota antilymphocyte globulin the aim was not to produce an antiserum with maximum potency on a comparative weight for weight basis with other drugs, but to produce a drug of optimal theraputic ratio designed to minimize the complications of administration while achieving additional beneficial immunosuppressive effects. Each dose was run intravenously through a central venous line or a large bore peripheral iv over a $6-\mathrm{hr}$ period.

In addition to antilymphocytic globulin, the patients received daily doses of steroids and cyclosporine. All patients received methylprednisolone at $15 \mathrm{mg} / \mathrm{kg}$ as the aortic cross clamp was released. Tapering dosages of methylprednisolone were given daily in two divided doses from $200 \mathrm{mg}$ to a $0.3 \mathrm{mg} / \mathrm{kg} /$ day maintenance dose.

Cyclosporine was begun on the day of transplant at a maintenance dose of $5 \mathrm{mg} / \mathrm{kg} /$ day for a serum creatinine less than $2.0 \mathrm{mg} \%$ and $2.5 \mathrm{mg} / \mathrm{kg} /$ day for a serum creatinine of greater than $2.0 \mathrm{mg} \%$. The cyclosporine dose was increased to maintain a whole blood level of 250-300 $\mathrm{ng} / \mathrm{ml}$, as determined by the high-performance liquid chromatography method. Laboratory analysis time to obtain cyclosporin levels at our institution was approximately $8 \mathrm{hr}$. Therefore, the decision to continue or discontinue polyclonal therapy after 7 days was made daily on the basis of cyclosporine level. If the cyclosporine level did not reach therapeutic levels upon the seventh postoperative day, antilymphocytic globulin therapy was continued. Total days of antilymphocytic therapy was equal in both groups and averaged 8 days of therapy. Azathioprine was begun after polyclonal prophylaxis at 1-2 $\mathrm{mg} / \mathrm{kg}$.

Daily white blood cell and platelet counts were obtained and recorded. Total lymphocyte counts were obtained in all patients and a therapeutic target of $200-300$ cells $/ \mathrm{mm}^{3}$ was considered adequate treatment. This immunosuppressive goal was reached equally and consistently in both groups, without differences between groups. $\mathrm{T}$-cell subsets were not obtained, since polyclonal therapy is not homogeneous between the two drugs or between different batches of the same drug. The level of specific antibodies is variable, and the resultant scatter in the T-cell subset levels would be so great as to render them meaningless. Antilymphocytic therapy was held for a total white blood cell count of less than $3.0 / \mathrm{mm}^{3}$. Patients received platelet transfusions for platelet counts of less than $50,000 / \mathrm{mm}^{3}$.
The patients underwent endomyocardial biopsy and right heart cardiac catheterization on the 10th to the 14th postoperative day. Following this, biopsies and cardiac catheterizations were obtained as dictated by the results of the first biopsy and the clinical postoperative course.

If severe histologic rejection, as defined by the criteria of Billingham [3], was identified at the time of biopsy, then antilymphocytic globulin was used for rescue therapy. Moderate rejection was treated with a steroid bolus of methylprednisolone at $30 \mathrm{mg} / \mathrm{kg} /$ dose/day for 3 days, and the biopsy was repeated in 10 days. If the moderate rejection persisted or rejection progressed, the patient received a second 3-day steroid treatment and was placed on either MALG or ATGAM at $15 \mathrm{mg} / \mathrm{kg} /$ dose for 7 days. The patients were then rebiopsied 10 days after the antilymphocytic globulin therapy was completed. Patients were considered to have had a rejection episode if they required treatment with either a steroid bolus or antilymphocytic globulin. Patients were considered to have had a second rejection episode if they had obtained a negative biopsy, prior to the second episode of rejection. Side effects of the drugs and differences in the rate and the severity of transplant organ rejection were compared.

Patients were closely followed for infectious complications in the postoperative period. Infections were categorized as to their severity and the etiology of the infection. Furthermore, the effects of the two drugs on diminishing the absolute white cell and lymphocyte counts were compared during drug administration and at 4 weeks following transplant. Results were analyzed using paired and unpaired $t$ tests, as well as analysis of variance. Confidence levels of greater than $95 \%(P<0.05)$ were considered significant.

\section{RESULTS}

The patient profiles of the 42 patients randomized to either MALG or ATGAM therapy are shown in Table 1. While the mean follow-up time and male to female ratios within each group did not differ significantly, there was a slightly but significantly decreased mean age in the MALG group as compared to the ATGAM group. No differences could be found demographically between these groups as to urgency of operation, pretransplant hemo-

\section{TABLE 1}

Patient Profiles Comparing MALG and ATGAM Groups

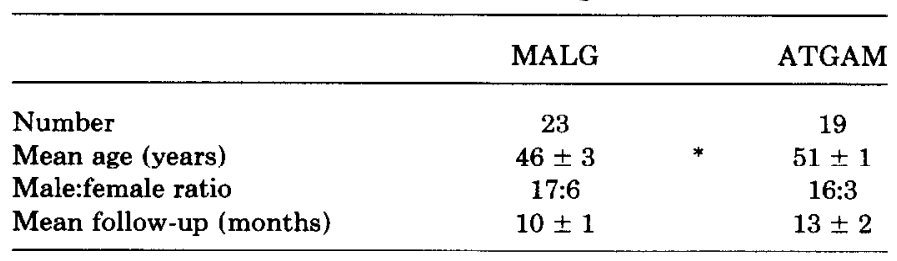

Note. Data are means \pm SEMs.

${ }^{*} P<0.05$ difference between MALG and ATGAM. 
dynamics, or underlying etiology of disease (ischemic vs idiopathic cardiomyopathy). There was no significant difference in the total number of daily ATGAM treatments versus the total number of MALG treatments per patient.

The use of MALG significantly delayed the onset of the first rejection episode from $22 \pm 3$ days post-transplant in the ATGAM group to $35 \pm 4$ days post-transplant in the MALG group $(P<0.03)$ (Table 2). Additionally, in the MALG group, four patients have been rejection free as compared to only one patient in the ATGAM group. The mean total rejection episodes for the MALG group were $1.5 \pm 0.2$ episodes for the follow-up period of the study as compared to $2.3 \pm 0.3$ episodes in the ATGAM group $(P<0.05)$ (Table 2). This translates to a significantly increased percentage of patients in the MALG group remaining free from rejection over time (Fig. 1). No difference in the severity of rejection was noted between groups. Finally, one patient in both of the groups died. The death in the MALG group was from an abrupt neurologic event, while the death in the ATGAM group was thought to have been from a severe acute dysrhythmia.

When the numbers of significant infections in both groups were compared, the ATGAM group had approximately twice the number of significant infections as the MALG group. $(11 / 19$ vs $6 / 23)(P<0.05)$ (Table 3$)$. The types of infection did not differ significantly between the two groups, and the spectrum of significant infections, defined as those requiring hospitalization, was representative of that seen in all immunosuppressed patients (i.e., bacterial, viral, pneumocystis). These infections included pneumonias and mediastinitis, but not urinary tract infections or wound infections, for which the patient did not require hospitalization.

No difference was found between the MALG and the ATGAM groups in terms of the total white blood count or total lymphocyte count, during treatment or 1 month following cardiac transplantation. Furthermore, no significant depression of these counts from normal was found 4 weeks following transplant.

There were no significant direct side effects noted in either group from the administration of the drugs, except one case of venous thrombophlebitis in the MALG group.

\section{TABLE 2}

Comparison of MALG and ATGAM Groups with Regard to Rejection of Transplant

\begin{tabular}{llll}
\hline & \multicolumn{1}{c}{ MALG } & \multicolumn{1}{c}{ ATGAM } \\
\hline $\begin{array}{lll}\text { Post-op days until first } \\
\text { rejection }\end{array}$ & $35 \pm 4$ & $*$ & $22 \pm 3$ \\
Mean rejection episodes/PT & $1.5 \pm 0.2$ & $*$ & $2.3 \pm 0.3$ \\
Deaths & 1 (stroke/seizure) & 1 (dysrhythmia) \\
Patients not rejecting & 4 & 1 \\
\hline
\end{tabular}

Note. Data are means \pm SEMs.

${ }^{*} P<0.05$ difference between MALG and ATGAM.

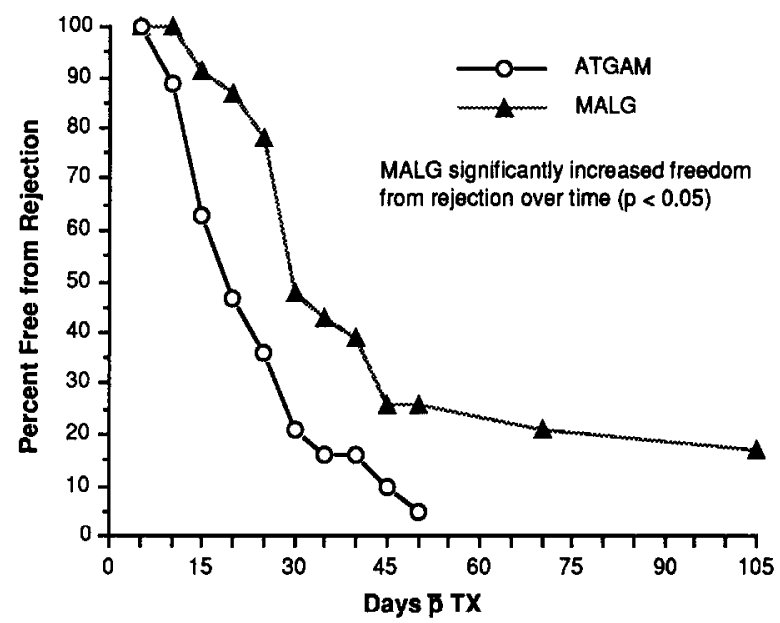

FIG. 1. Comparison of MALG and ATGAM groups showing a significantly increased freedom from rejection over time with MALG treatment.

\section{DISCUSSION}

Polyclonal heterologous antibody sera directed against human immune cells has been used in transplantation for over 20 years [15]. The first clinical study to compare antithymocyte vs antilymphocyte therapy was published in 1972 from the Stanford University group [6]. This prospective nonrandomized study compared 26 cardiac transplant patients operated upon between 1968 and 1971 and administered either antithymocyte or antilymphocyte globulin therapy. These patients were transplanted before

\section{TABLE 3}

Comparison of MALG and ATGAM Groups with Regard to Infections and Suppression of Immunologic Active Cells

\begin{tabular}{|c|c|c|}
\hline & MALG & ATGAM \\
\hline Significant infections & $6 / 23(26 \%)$ & * $11 / 19(58 \%)$ \\
\hline $\begin{array}{l}\text { Total white blood count } \\
\text { ( } 4 \text { weeks post- } \\
\text { transplant) }\end{array}$ & $8.4 \pm 2.4$ & $9.0 \pm 3.0$ \\
\hline $\begin{array}{l}\text { Total lymphocyte count } \\
\text { (4 weeks post- } \\
\text { transplant) }\end{array}$ & $15 \pm 3$ & $9 \pm 3$ \\
\hline \multicolumn{3}{|l|}{ Type of infection } \\
\hline Bacterial & $\begin{array}{l}3 \text { (Pneumonia) } \\
\text { (Colitis) } \\
\text { (Mediastinitis) }\end{array}$ & $\begin{array}{l}7 \text { (Groin wound infection) } \\
\text { (Sternal infection) } \\
\text { (Pneumonia } \times 3 \text { ) } \\
\text { (Catheter sepsis) } \\
\text { (Urosepsis) }\end{array}$ \\
\hline Viral & $\begin{array}{r}2 \text { (Esophagitis) } \\
\text { (Pneumonia) }\end{array}$ & $\begin{array}{l}3 \text { (Mediastinitis) } \\
\text { (Hepatitis) } \\
\text { (Pneumonia) }\end{array}$ \\
\hline Pneumocystis & 1 (Pneumonia) & 1 (Pneumonia) \\
\hline
\end{tabular}

Note. Data are means \pm SEMs.

${ }^{*} P<0.05$ difference between MALG and ATGAM. 
the cyclosporine era. Those patients in the ALG group demonstrated their first episode of acute rejection on the 7th postoperative day, while those treated with ATG experienced rejection on Day 12. This was not a significant difference. However, patients treated with antithymocyte globulin experienced a markedly increased incidence of viral and fungal infections, which led to the death of 5 of these patients. The optimal mode of antilymphocytic therapy could not be determined from this study.

In the cyclosporine era, while there have been comparisons of polyclonal antibody therapy ( $\Lambda$ TGAM) vs monoclonal therapy (OKT3) [9, 13], there has not been a comparison in the cardiac transplant patient population between the two most commonly used modes of antilymphocytic therapy (MALG vs ATGAM).

MALG has been shown to be effective in many different randomized trials, as has ATGAM. Both have been shown to significantly improve both organ and patient survival following renal transplantation $[4,10,11,16]$ and cardiac transplantation $[1,8,17]$.

The reasons for the significant delay in the first rejection episode in our patients treated with MALG as opposed to ATGAM may lie in specific immune differences in each of the polyclonal sera.

Both MALG and ATGAM are monomeric (equine) IgG antibodies that possess immunologic activity against normal peripheral human lymphocytes, mainly circulating T-cells [14]. Antilymphocytic therapy used as prophylaxis in cardiac transplant recipients has been demonstrated in previous studies to produce suppression of the inducerhelper T-cells (OKT4) up to 10 days following cardiac transplantation [8]. Suppressor-cytotoxic T-cells (OKT8) are also suppressed in this patient population, and therefore, the ratio of the two (OKT4/OKT8), which is used as an index of immunosuppression, is unchanged. Total T-cells (OKT11) are significantly suppressed for up to 20 days after surgery with antilymphocytic therapy. This supports the findings of other investigators [2], who found an overall reduction in total T-cells (OK'T11), as well as a selective action of antithymocyte globulin in suppressing inducer-helper cells (OKT4). Neither our institution [7] nor others [12] have found monitoring these subpopulations to be predictive of rejection. Therefore specific lymphocyte populations were not followed in our preliminary study reported here, and no alteration in total lymphocyte count was found in either group.

Lymphocytes for MALG preparation were originally obtained from a single healthy human donor and have been grown continuously in culture. These lymphoblasts, when used for immunizations in the horse, result in a polyclonal antibody sera with a uniform potency and may minimize variations in the individual doses of MALG. ATGAM is prepared in much the same way, but ATGAM is derived from donated human thymus tissue and may be of variable immunologic potency.

Preparation of both MALG and ATGAM includes absorption of human red cell stroma and platelets, stabili- zation, removal of pyrogens, and reduction of the titer of reactive antibodies. However, thymus tissue may also have other tissues carried with it and may elicit antibodies, in the immunized horses, to human cell lines, which, despite purification, may persist in the drug which is administered to human transplant recipients. This may lead to increased side effects which can include thrombocytopenia, anemia, fever, anaphylatic reactions, and deposition of protein in renal basement membranes [4]. Also it may possibly reduce the effectiveness of ATGAM or increase the variability from lot to lot.

As can be seen from our results despite the optimal and equal immunosuppression acheived by both modalities of therapy, MALG significantly delayed the first rejection episode compared to ATGAM, when used as early prophylaxis following cardiac transplantation. Additionally there was decreased rejection during the follow-up period for the MALG group compared to the ATGAM group. Finally, the significant infection rate with MALG appears to be decreased compared to that of the ATGAM group. While the somewhat increased incidence of infection in the ATGAM group may not be explained entirely by specific immunologic differences between MALG and ATGAM, the increased incidences of rejection in the ATGAM group, leading to multiple episodes of repeated intense steroid therapy or further 7-day courses of antilymphocytic therapy, may have led to overimmunosuppression and an increased infection rate in the ATGAM group. Although the severity of the infections was not different between the two groups, and no patient in either group succumbed to infectious complications, the increased incidence of infection requiring hospitalization in the ATGAM group was a significant finding.

Our data suggest that MALG is the more favorable polyclonal antibody sera for early prophylaxis in cardiac transplantation patients. This study, however, involved only short term follow-up, and further definition of the immunologic differences hetween these two therapies and long-term differences between these two groups merit investigation. One might assume that increasing the dose of ATGAM to higher levels, in an attempt to reduce rejection episodes, could severely increase the infection rate.

\section{ACKNOWLEDGMENTS}

We thank Upjohn Pharmaceutical (Kalamazoo, MI) and the University of Minnesota, particularly Dr. Richard Condie, for their assistance.

\section{REFERENCES}

1. Barnhart, G. R., Goldman, M. H., Hastillo, A., et al. Comparison of immunosuppression therapy following heart transplantation: Pretransfusion/azathioprine/ATG/prednisone versus cyclosporine/prednisone. J. Heart Transplant. 4: 381, 1985.

2. Carver, M., Thomas, F., Deepe, R., et al. Selective action of rabbit anti-lhymocyte globulin (RATG) on OKT4 positive T-cells subset in rhesus monkeys. Transplant. Proc. 15: 627, 1983.

3. Caves, P. K., Billingham, M. F., Stinson, E. B., et al. Serial trans- 
venous biopsy of the transplanted human heart-improved management of acute rejection episodes. Lancet 1: 821, 1974.

4. Condie, R. M., Waskosky, K. E., Hall, B. L., et al. Efficacy of Minnesota antilymphoblast globulin (MALG) in renal transplantation: A multicenter, placebo-controlled, prospective, randomized, double-blind study. Proceedings of the 10th International Congress of the Transplantation Society, August 1984. Transplant. Proc. 17: 1304,1985 .

5. Deeb, G. M., Kolff, J., McClurken, J. B., et al. Antithymocyte $\gamma$ globulin, low dosage cyclosporine, and tapering steroids as an immunosuppressive regimen to avoid early kidney failure in heart transplantation. J. Heart Transplant. 6: 79, 1987.

6. Griepp, R., Stinson, E., Dong, E., Jr., Phillips, R., and Morrell, R. Use of antithymocyte globulin in human heart transplantation. Cardiac Transplant. 45-46: I117, 1971.

7. Hanson, C. A., Bolling, S. F., Stoolman, L. M., et al. Cytoimmunologic monitoring (CIM) in cardiac transplantation. $J$. Heart Transplant. 7: 68, 1988.

8. Kawaguchi, A., Szentpetery, S., Mohanakumar, T., Barnhart, G. R., and Lower, R. R. Effects of prophylactic rabbit antithymocyte globulin in cardiac allograft recipients treated with cyclosporine. J. Heart Transplant. 6: 214, 1987.

9. Kormos, R. L., Herlan, D. B., Curran, M., Hardesty, R. L., Armitage, J., and Griffith, B. P. Monoclonal vs polyclonal antibody therapy for prophylaxis against rejection following cardiac transplantation. J. Heart Transplant. 7: 82, 1988.

10. Najarian, J. S., Simmons, R. L., Condie, R. M., et al. Seven years' experience with antilymphoblast globulin for renal transplantation from cadaver donors. Ann. Thorac. Surg. 184: 352, 1976.

11. Novick, A. C., Braun, W. E., Steinmuller, D., et al. A controlled randomized double-blind study of anyilymphoblast globulin in cadaver renal transplantation. Transplantation 35: 175, 1983.

12. O'Toole, C. M., Maher, P., Spiegelhalter, D. J., Walker, J. R., Stovin, P., Wallwork, J., and English, T. A. H. Rejection or infection predictive value of $\mathrm{T}$-cell subject ratio, before and after heart transplantation. J. Heart Transplant. 4: 518, 1985.

13. Renlund, D. G., O'Connell, J. B., Gilbert, E. M., Burton, N. A., Jones, K. W., Karwande, S. V., Menlove, R. L., Gay, W. A., Jr., and Bristow, M. R. Comparison of OKT3-versus ATG-based early prophylaxis: Decreased rejection and corticosteroid use with OKT3. J. Heart Transplant. 7: 69, 1988.

14. Schmidtke, J. R., Ferguson, R. M., Beierle, M. J., et al. The clinical significance of pre- and post-transplant immunologic monitoring. Transplant. Proc. 9(1): 350, 1979.

15. Starzl, T. E., Marchioro, T. L., Porter, K. A., Iwaski, Y., and Cerilli, G. J. Use of heterologous antilymphoid agents in canine renal and liver homotransplantation and in human renal transplantation. Surg. Gynecol. Obstet. 124: 301, 1967.

16. Turcotte, J. G., Reduska, N. J., Naines, R. F., et al. A clinical trial of antithymocyte globulin in renal transplant recipients. Arch. Surg. 106: 484, 1973.

17. Yacoub, M., Alvizotos, P., Khaghani, A., et al. Cyclosporine and antithymocyte globulin with or without low-dose steroids for immunosuppression of cardiac transplant patients. Transplant Proc. 15: $1247,1983$. 